\title{
Avaliação da eficácia da microabrasão no tratamento de manchas de fluorose: revisão de literatura
}

\author{
Evaluation of the efficacy of microabrasion in the treatment of fluorosis stains: literature review \\ Evaluación de la eficacia de la microabrasión en el tratamiento de las manchas de fluorosis: revisión da literatura \\ Pedro Paulo Aguiar Santos CAVALCANTI ${ }^{1}$ \\ Maria Eduarda Arruda de LUCENA ${ }^{1}$ \\ Hian Carvalho SOUZA 1 \\ Eloiza Leonardo de MELO² \\ Jéssica Meirinhos MIRANDA2 \\ Lara Marques Magalhães MORENO2 \\ Marleny Elizabeth Márquez de Martínez GERBI ${ }^{4}$ \\ Maria Regina Almeida de MENEZES ${ }^{3}$ \\ ${ }^{1}$ Graduando (a) em Odontologia - FOP-UPE, Faculdade de Odontologia de Pernambuco, FOP Univ. de Pernambuco UPE, 54756-220 Camaragibe - PE, Brasil \\ ${ }^{2}$ Doutoranda em Odontologia - FOP-UPE, Faculdade de Odontologia de Pernambuco, FOP Univ. de Pernambuco UPE, 54756-220 Camaragibe - PE, Brasil \\ ${ }^{3}$ Professora Adjunta de Dentística - FOP-UPE, Faculdade de Odontologia de Pernambuco, FOP Univ. de Pernambuco UPE, 54756-220 Camaragibe - PE, Brasil \\ ${ }^{4}$ Professora de Implantodontia - FOP-UPE, Faculdade de Odontologia de Pernambuco, FOP Univ. de Pernambuco UPE, 54756-220 Camaragibe - PE, Brasil
}

\section{Resumo}

Introdução: A fluorose dentária é uma patologia que afeta os elementos dentários sendo desencadeada pela presença em excesso de fluoreto, e manifestando-se ao nível do esmalte dentário na forma de manchas e/ou defeitos anatómicos. Objetivo: Avaliar a eficácia da microabrasão no tratamento da fluorose e verificar a aplicabilidade desta técnica nos dias atuais, ratificando a importância da microabrasão como um tratamento estético minimamente invasivo. Materiais e Métodos: Foram realizadas buscas nas bases de dados Google Acadêmico, Scielo e PubMed/MEDLINE através da inserção dos termos "Enamel Microabrasion AND Fluorosis Dental". Os critérios de inclusão foram: Casos clínicos, publicados de 2015 à 2019, escritos na língua portuguesa, espanhola e inglesa. Os critérios de exclusão foram os artigos que não estavam nas bases de dados pré-definidas, artigos que não possuíam textos disponibilizados na íntegra e artigos em outras línguas das que foram definidas. Os dados foram extraídos e tabelados e posteriormente foi realizada a análise qualitativa dos dados. Resultados: Foram identificados 1.411 artigos nas bases de dados, sendo 1330 na base Google Acadêmico, 2 na base scielo, 79 na base PubMed/MEDLINE e foram excluídos 946 artigos de acordo com os critérios de inclusão e exclusão. Conclusão: Apesar de diminuir a espessura do esmalte, a microabrasão como técnica para o tratamento permite a resolução de problemas estéticos de maneira minimamente invasiva, com alto grau de satisfação pelos pacientes e pelos profissionais e ainda com baixo custo. Além disso, favorece os pacientes por trazer resultados imediatos e permanentes com mínima e imperceptível perda de esmalte.

Descritores: Fluorose Dentária; Microabrasão do Esmalte; Esmalte Dentário.

\section{Abstract}

Introduction: Dental fluorosis is a pathology that affects dental elements being unleashed by the presence of fluoride in excess, manifesting at the level of tooth enamel in a form of stains and/or anatomic defects. Objective: Evaluate the efficiency of microabrasion in fluorosis treatment and verificate the applicability of these technique in actual days, ratifying the importance of microabrasion as a aesthetic treatment with minimal invasion. Materials and methods: Were made researches in bases of data Scholar Google, Scielo and PubMed/MEDLINE through the insertion of texts "Enamel Microabrasion AND Fluorosis Dental". The inclusion criteria were: Clinical cases published from 2015 to 2019, written in portuguese, spanish and english language. The datas were extracted and tabulated and posteriorly made a qualitative analysis of data. Results: Were identified 1.411 articles in a base of data, being 1.330 in Scholar Google base, 2 in Scielo base, 79 in PubMed/MEDLINE base and were excluded 946 articles according the inclusion and exclusion criterias. Conclusion: Despite decreasing the enamel thickness, the microabrasion as a technique for treatment allows the resolution of aesthetics problems in a minimal invasive way, with high grade of satisfaction by the patients and professionals and even with a low cost.

Descriptors: Fluorosis, Dental; Enamel Microabrasion; Dental Enamel.

\section{Resumen}

Introducción: la fluorosis dental es una patología que afecta a los elementos dentales, desencadenada por la presencia del exceso de fluoruro, y se manifiesta al nivel del esmalte dental en forma de manchas y / o defectos anatómicos. Objetivo: evaluar la eficacia de la microabrasión en el tratamiento de la fluorosis y verificar la aplicabilidad de esta técnica en los días actuales, ratificando la importancia de la microabrasión como un tratamiento estético mínimamente invasivo. Materiales y Métodos: se realizaron búsquedas en las bases de datos Google Académico, Scielo y PubMed / MEDLINE a través de la inserción de los términos "Enamel Microabrasion AND Fluorosis Dental". Los criterios de inclusión fueron: casos clínicos, publicados desde 2015 hasta 2019, escritos en portugués, español e Inglés. Los criterios de exclusión fueron los artículos que no estaban en las bases de datos predefinidas, artículos que no poseían textos disponibles en su totalidad y artículos en otras lenguas de las que se definieron. Los datos fueron extraídos y tabulados, y posteriormente se realizó el análisis cualitativo de los datos. Resultados: se identificaron 1.411 artículos en las bases de datos, siendo 1330 en la base Google Académico, 2 en la base scielo, 79 en la base PubMed / MEDLINE y se excluyeron 946 artículos de acuerdo con los criterios de inclusión y exclusión. Conclusión: aunque de disminuir el espesor del esmalte, la microabrasión como técnica para el tratamiento permite la resolución de problemas estéticos de manera mínimamente invasiva, con bajo costo y presenta un alto grado de satisfacción para los pacientes y para los profesionales. Además, favorece a los pacientes por traer resultados inmediatos y permanentes con mínima e imperceptible pérdida de esmalte.

Descriptores: Fluorosis Dental; Microabrasión de Esmalte; Esmalte dental.

INTRODUÇÃO

Os padrões impostos pela sociedade estão cada vez mais incitando desejos no ser humano, dentre eles, a questão da busca pelos dentes perfeitos, o que vem se tornando uma meta comum entre muitos, e um sorriso harmônico seja em proporção, forma, alinhamento e cor se tornou sinônimo de bemestar e consequentemente, saúde ${ }^{1}$. A partir disso os pacientes buscam o Cirurgião-Dentista (CD) a fim de suprir tais necessidades, projetando tais desejos no profissional, um dos mais comuns na prática clínica é a resolução de manchas, mais precisamente as relacionadas a fluorose ${ }^{1}$.

A fluorose é resultante de um distúrbio que ocorre na odontogênese, precisamente durante a amelogênese, e é caracterizada pelo aumento da porosidade em superfície, principalmente em esmalte, tornando-o opaco ${ }^{1}$. Ocorre quando há ingestão contínua de quantidades de flúor acima da dose ideal 
durante a odontogênese, causando manchas imperceptíveis ou manchas muito brancas, amareladas ou amarronzadas, que podem representar um problema estético; nos casos muito graves, pode haver perda quase total da superfície de esmalte, com mudanças na anatomia dentária, sendo que a intensidade da sua manifestação é diretamente associada à quantidade de flúor ingerido ${ }^{2}$.

Segundo Chankanka ${ }^{3}$ em uma pesquisa sobre aparência do esmalte dentário, notou-se que a fluorose muito leve e leve tem pouco ou nenhum efeito na qualidade de vida relacionada à saúde bucal (QVRSB - Qualidade de vida relacionada à saúde bucal), diferentemente nos casos de fluorose grave, que pode ser observado um impacto negativo.

No passado era comum que os dentes que apresentassem anomalias de cor fossem tratados com desgastes dentários objetivando a remoção do tecido comprometido, seguido de restauração com materiais diretos ou indiretos a fim de restabelecer a estética do elemento dentário. No entanto, esses materiais necessitam de constante substituição, dando a técnica a característica de não longevidade, sendo um fator desfavorável ${ }^{4}$.

Devido ao momento atual que a Odontologia vive, onde se preconiza procedimentos minimamente invasivos, este trabalho tem como objetivo resgatar um procedimento empregado há anos, por apresentar resultados imediatos e duradouros, sem que haja um desgaste em excesso do esmalte e verificar a eficácia da microabrasão no tratamento da fluorose.

\section{MATERIAL E MÉTODO}

Foram realizadas buscas nas bases de dados Google Acadêmico ,Scielo e PubMed/MEDLINE através da inserção dos termos "Enamel Microabrasion AND Fluorosis Dental". Os critérios de inclusão foram: Casos clínicos, publicados de 2015 a 2019, escritos na língua portuguesa, espanhola e inglesa. Os critérios de exclusão foram os artigos que não estavam nas bases de dados pré-definidas, artigos que não possuíam textos disponibilizados na íntegra e artigos em outras línguas das que foram definidas. Os dados foram extraídos e tabelados e posteriormente foi realizada a análise qualitativa dos dados. O processo de busca foi executado em três fases através da busca de títulos, análise de resumos e identificação de artigos de texto completo.

\section{RESULTADOS}

A pesquisa com os descritores selecionados resultou em 1.411 artigos, sendo 1330 no Google Acadêmico, 2 no Scielo e 79 no PubMed/MEDLINE. Após a leitura do título e resumo e foram excluidos 946 artigos. Foram selecionados para leitura na íntegra 147 artigos, sendo excluídos 116 artigos (não falavam especificamente da técnica de microabrasão), 9 estavam duplicados e 3 eram livros.
O resultado final da revisão foram 10 publicações, sendo todos classificados como artigos científicos (Figura 1).

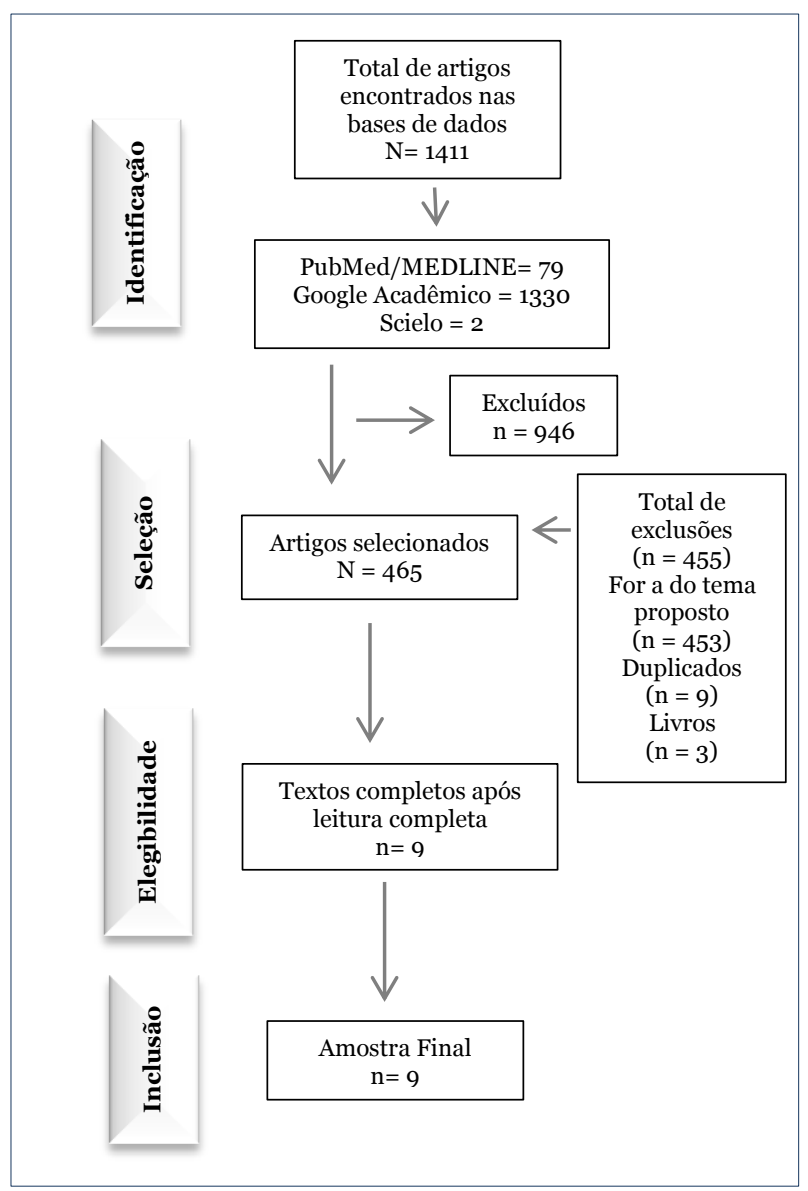

Figura 1: Fluxograma de seleção dos artigos

A Tabelas 1 e 2 mostram as características dos estudos incluídos, tais como autor, localização do estudo, amostra, objetivo e conclusão.

Tabela 1. Características dos artigos selecionados.

\begin{tabular}{|c|c|}
\hline Autor/Ano & Local \\
\hline Hermes $^{1}, 2013$ & Universidade de Santa Cruz do Sul - Rio Grande do Sul \\
\hline Conclusão & 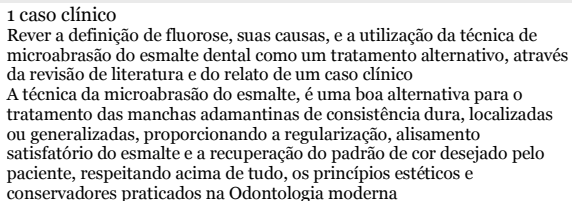 \\
\hline Autor/Ano & Local \\
\hline $\begin{array}{l}\text { Prado J } \\
2018\end{array}$ & Universidade Federal do Piauí. \\
\hline Amostra & 1 caso clínico \\
\hline Objetivo & $\begin{array}{l}\text { Mostrar um caso clínico de tratamento de fluorose de esmalte resolvido } \\
\text { com microabrasão, usando o ácido fosforico } \\
\text { A microabrasão dentária é uma boa alternativa para tratamento estético } \\
\text { da fluorose dentária, porque desgasta pouca estrutura dentária, } \\
\text { restabelece a estética, não causa danos à polpa nem aos tecidos } \\
\text { periodontais, requer pouco tempo de tratamento, não necessita de } \\
\text { preparo de cavitário, nem de material restaurador e é de baixo custo }\end{array}$ \\
\hline Autor & Local \\
\hline Agostin & Universidade Federal de Minas Gerais \\
\hline Amostra & $\begin{array}{l}\text { Base de dados com artigos sobre o tema da fluorose dentária de } 2001 \text { a } \\
2010 \\
\text { Este trabaho teve como objetivo fazer uma revisão de literatura narrativa } \\
\text { sobre os estudos da fluorose dentária na década de } 2001 \text { a } 2010\end{array}$ \\
\hline Conclusão & $\begin{array}{l}\text { Nos casos de fluorose leve, que são as formas mais prevalentes, o } \\
\text { tratamento mais indicado é a combinação de técnicas de microabrasão do } \\
\text { esmalte e clareamento dental, por serem considerados os tratamentos } \\
\text { menos invasivos na literatura para a diminuição dos efeitos da fluorose }\end{array}$ \\
\hline Autor/Ano & \\
\hline Bağlar s & $\begin{array}{l}\text { Universidade e Faculdade de Odontologia Kırıkkale, Kırıkkale, } \\
\text { Turquia }\end{array}$ \\
\hline Amostra & $\begin{array}{l}\text { Quarenta dentes incisivos superiores extraídos foram utilizados neste } \\
\text { estudo }\end{array}$ \\
\hline Objetivo & $\begin{array}{l}\text { Produzir um protótipo de pasta de microabrasão para o tratamento de } \\
\text { manchas de fluorose e examinar os efeitos da pasta na estrutura e cor } \\
\text { dentária in vitro }\end{array}$ \\
\hline Conclusão & $\begin{array}{l}\text { O estudo demonstrou que a pasta protótipo é uma opção de tratamento } \\
\text { viável para a fluorose dentária }\end{array}$ \\
\hline
\end{tabular}


Tabela 2. Características dos artigos selecionados

\begin{tabular}{|c|c|}
\hline Autor/Ano & Local \\
\hline Di Giovanni, 2018 & Universidade de Zurique, Zurique, Suíça \\
\hline Amostra & 348 pacientes \\
\hline Conclusão & $\begin{array}{l}\text { Fluorose dentária tem implicações consideráveis na qualidade de vida } \\
\text { dos pacientes. O presente estudo avalia a eficácia comparativa das } \\
\text { várias intervencốes para o tratamento do esmalte fluoroso } \\
\text { Com base nas evidências limitadas existentes, a infiltração de resina } \\
\text { parece ser o tratamento mais promissor para a fluorose dentária, } \\
\text { seguido pelo branqueamento e microabrasão }\end{array}$ \\
\hline Autor/Ano & Local \\
\hline Gupta $^{8}, 2017$ & $\begin{array}{l}\text { Faculdade de Ciências Odontológicas, Universidade SGT, } \\
\text { Gurgaon, Haryana, Índia }\end{array}$ \\
\hline Amostra & 90 crianças. \\
\hline Conclusão & $\begin{array}{l}\text { O objetivo do estudo foi avaliar e comparar a eficácia de técnicas } \\
\text { minimamente invasivas para a remoção de manchas de fluorose } \\
\text { dentária em crianças in vivo } \\
\text { Técnicas de branqueamento e microabrasão podem ser consideradas } \\
\text { alternativas interessantes para as opções de tratamento cirúrgico } \\
\text { convencional }\end{array}$ \\
\hline Autor/Ano & Local \\
\hline Meireles et al. 9,2018 & Universidade Federal da Paraíba, Paraíba \\
\hline Amostra & 70 indivíduos \\
\hline Conclusão & $\begin{array}{l}\text { Avaliar o efeito na qualidade de vida relacionada à saúde bucal } \\
\text { (QVRSB) de dois protocolos de tratamento para fluorose dentária, em } \\
\text { indivíduos inclúdos em um ensaio clínico randomizado. } \\
\text { Concluiu-se que o tratamento com microabrasão melhorou a QVRSB de } \\
\text { indivíduos vivendo em uma área de fluorose endêmica } \\
\text { independentemente da associação com o clareamento caseiro }\end{array}$ \\
\hline Autor/Ano & Local \\
\hline Oliveira et al.10, 2014 & Universidade Federal de Minas Gerais \\
\hline Amostra & 1 caso clínico \\
\hline Conclusão & $\begin{array}{l}\text { Relatar um caso clínico apresentando a técnica de microabrasão de } \\
\text { esmalte como opção eficaz de tratamento de fluorose dentária } \\
\text { moderada } \\
\text { A microabrasão com ácido clorídrico } 6 \% \text { e carbeto de silício é uma } \\
\text { técnica eficaz para remoção de manchas de fluorose moderada, além de } \\
\text { favorecer a estética do sorriso e poder contribuir para a melhora da } \\
\text { autoestima e do convívio social }\end{array}$ \\
\hline Autor/Ano & Local \\
\hline Pini et al. ${ }^{11} 20$ & UNICAMP, Piracical \\
\hline Amostra & Revisão sistemática sobre a técnica da microabrasão \\
\hline Objetivo & $\begin{array}{l}\text { A presente revisão de literatura tem por objetivo discutir as indicações e } \\
\text { aspectos clínicos e científicos da técnica de microabrasão, bem como } \\
\text { seus efeitos na superfície do esmalte }\end{array}$ \\
\hline Conclusão & $\begin{array}{l}\text { Os resultados laboratoriais e clínicos apresentados nesses artigos } \\
\text { apóiam o uso da microabrasão do esmalte como primeira opção de } \\
\text { tratamento para pacientes que preferem uma abordagem menos } \\
\text { invasiva }\end{array}$ \\
\hline
\end{tabular}

\section{DISCUSSÃO}

Em todos os trabalhos analisados houve um consenso que há diferentes apresentações e características das lesões de mancha branca na estrutura dentária, onde as mesmas podem apresentar etiologias variadas ${ }^{2}$. Esta constatação é relevante, pois trás a importância de um correto diagnóstico para a implementação de um plano de tratamento eficaz para cada tipo de mancha branca, principalmente se esta mancha ocorrer em decorrência de um processo carioso, a avaliação se a doença cárie está ativa deve ser levada em consideração para a redução ou até a estagnação de lesões de cárie.

O tratamento dessas manchas tem sido uma demanda recorrente nos consultórios odontológicos, devido a atual busca pelo sorriso padrão de perfeita forma, proporção, cor e alinhamento. E tal resolução repercute diretamente na autoestima do paciente, fazendo com que o CD fique diante da decisão de eleger o procedimento mais adequado, que não apenas solucione tais alterações de coloração, mas que também mantenha a estrutura dentária da melhor maneira possível ${ }^{1}$. No caso de lesões de mancha branca ativa, o paciente deve ser orientado em relação aos cuidados com sua higiene bucal, para logo após serem realizados os procedimentos de adequação do meio bucal.

Meireles et al. ${ }^{9}$ concluíram em seu trabalho que a microabrasão reduziu a QVRSB dos pacientes que viviam em uma área de fluorose endêmica, independente da aplicação do clareamento caseiro, entretanto Oliveira et al. ${ }^{10}$ relatam em seu estudo que a indicação da técnica de microabrasão deve ser levada em consideração que pode ter uma extensão superficial ou ser mais profunda, concluindo que em alguns casos o procedimento de microabrasão pode produzir apenas discreta melhora no aspecto clínico, o que deve ser discutido com o paciente.

Outras formas de tratamento da fluorose dentária como facetas estéticas e coroas protéticas, mas autores como Hermes ${ }^{1}$, elegem a microabrasão por ser eficaz e segura, conservadora e estética, justificando que esta técnica tem sido eficaz quanto a remoção de irregularidades e manchas de esmalte dental $^{1}$, efetividade esta que tem sido demonstrada desde sua introdução. Eles ainda classificam, também, a microabrasão como uma técnica menos invasiva, a qual não causa nenhum desconforto durante ou após o procedimento ${ }^{12,13}$.

Mesmo que haja distintas técnicas e métodos para serem utilizados, o desgaste do esmalte apresenta características equivalentes, com variação apenas na quantidade do que foi desgastado de acordo com o tempo de microabrasão, método aplicado e o material utilizado ${ }^{1}$. A microabrasão de esmalte também está indicada junto ao clareamento dental por se tratar de um procedimento pouco invasivo, e conjuntamente alcançam maior diminuição dos efeitos da fluorose ${ }^{5}$.

Uma vantagem no pós-operatório segundo Sundfeld et al. ${ }^{14}$ é que com até $10 \%$ de espessura de desgaste do esmalte por microabrasão, não há prejuízo a estrutura remanescente e relação com a sensibilidade dental. Hermes ${ }^{1}$ também relata que a microabrasão além de não causar sensibilidade pósoperatória, apresenta resultado imediato, não causa danos à polpa e aos tecidos periodontais, o procedimento envolve pouco tempo e é uma técnica de baixo custo.

De forma sucinta os autores descrevem o processo como de início o isolamento absoluto seguido da aplicação de ácido clorídrico a $18 \%$ ou uma pasta para abrasão químico-mecânica ambos misturados a um abrasivo, usualmente é escolhido a pedra-pomes. Com um contra-ângulo com redutor de velocidade começa a remover a camada superficial, com cautela, até encontrar o esmalte normal, é importante ressaltar que ao final de cada período os dentes sejam lavados com água abundante. Posteriormente é realizado o polimento do esmalte dentário com disco de feltro ou borrachas abrasivas de granulação fina e pasta para polimento ${ }^{1,5,9}$.

\section{CONCLUSÃO}

A microabrasão é uma técnica indicada para o tratamento da fluorose dentária, visto que permite a resolução de problemas estéticos de maneira minimamente invasiva, tornando-se uma boa alternativa no tratamento de manchas localizadas ou 
generalizadas, promovendo a regularização e alisamento satisfatório do esmalte e também, a recuperação de um padrão de cor, inicialmente desejado pelo paciente, porém respeitando os princípios estéticos e conservadores da Odontologia moderna.

\section{REFERÊNCIAS}

1. Hermes SR. Microabrasão do esmalte dental para tratamento de fluorose. RGO Rev gaúch odontol. 2013; 61 (supl 1):427-33.

2. Prado Júnior RR, Ribeiro RC, Brito AC, Lopes TSP. Microabrasão como tratamento de esmalte fluorótico. RGO Porto Alegre. 2008;56(2):21-6.

3. Chankanka O, Levy SM, Warren JJ, Chalmers JM. A literature review of aesthetic perceptions of dental fluorosis and relationships with psychosocial aspects/oral health-related quality of life. Community Dent Oral Epidemiol. 2010; 38(2):97-109.

4. Calixto LR, Galafassi G, Alves MR, Mandarino F. Tratamento de manchas dentais: Clareamento e Microabrasão. Caderno Científico, Estética. 2007:20-2.

5. Agostini M. Fluorose Dentária: uma revisão de literatura [monografia]. Belo Horizonte: Universidade Federal de Minas Gerais, Curso de Especialização em Atenção Básica em Saúde da Família; 2011.

6. Bağlar S, Çolak H, Hamidi MM - Novel microabrasion paste. J Esthet Restor Dent. 2015; 27(5):258-66.

7. Di Giovanni T, Eliades T, Papageorgiou SN. Interventions for dental fluorosis: A systematic review. J Esthet Restor Dent. 2018;30(6):502-8.

8. Gupta A, Dhingra R, Chaudhuri P, Gupta A. A comparison of various minimally invasive techniques for the removal of dental fluorosis stains in children. J Indian Soc Pedod Prev Dent. 2017;35(3):260-68.

9. Meireles SS, Goettems ML, Castro KS, Sampaio FC, Demarco FF. Dental fluorosis treatment can improve the individuals' OHRQoL? Results from a randomized clinical trial. Braz Dent J. 2018;29(2):109-16.

10. Oliveira LMX, Novaes Junior JB, Barreiros ID, Paiva SM, Martins CC. Tratamento de fluorose dentária moderada com a técnica de microabrasão de esmalte com ácido clorídrico $6 \%$ e carbeto de silício: relato de caso clínico. Arq Odontol. 2014;50(3):142-48.

11. Pini NI, Sundfeld-Neto D, Aguiar FH, Sundfeld RH, Martins LRM, Lovadino JR et al. Enamel microabrasion: An overview of clinical and scientific considerations. World J Clin Cases. 2015;3(1):34-41.

12. Pinto MM, Guedes CC, Motta LJ, Bussadori SK. Alternativa estética para manchas de fluoroseempregando técnicas de microabrasão e clareamento em adolescents. Rev Assoc paul cir dent. 2009;63(2):130-33.

13. Mendonça AAM, Ponciano ACR, Fregonesi F, Porto CLA, Campos EA. Microabrasão como alternativa estética - relato de caso clínico. JBC j. bras. clin. odontol. Integr. 2006;10(54):200-3.

14. Sundfeld RH, Komatsu J, Russo M, Holland Junior C, Castro MAM, Quintella LPAS et al. Remoção de manchas no esmalte dental: estudo clínico e microscópico. Rev bras odontol. 1990;47(3):29-34.

\section{CONFLITO DE INTERESSES}

Os autores declaram não haver conflitos de interesse.

\section{AUTOR PARA CORRESPONDÊNCIA}

\section{Pedro Paulo Aguiar Santos Cavalcanti}

Faculdade de Odontologia de Pernambuco

Av. General Newton Cavalcanti, 1650

54756-220 - Tabatinga - Camaragibe - PE, Brasil

E-mail: pedropauloaguiar@icloud.com 